\title{
ARTICLE OPEN \\ Mitigating the contrail cirrus climate impact by reducing aircraft soot number emissions
}

\author{
Ulrike Burkhardt (iD) ${ }^{1}$, Lisa Bock (iD) and Andreas Bier (D)
}

Contrail cirrus are a major component of the climate forcing due to air traffic. For a given contrail cirrus cover, ice water content and ice crystal shape, their impact on radiation is dependent on the number and size of ice crystals. Here we use a global climate model to study the impact of a reduction in initially formed ice crystal numbers, as may be caused by reduced soot number emissions. We find that for reduced initial ice crystal numbers the ice water content is decreased and ice crystal sizes increased, leading to a reduction in contrail cirrus optical depth and doubling the fraction of contrail cirrus that cannot be detected by satellite remote sensing. Contrail cirrus lifetimes and coverage are strongly reduced leading to significant reductions in contrail cirrus radiative forcing. The global climate impact of contrail cirrus is nonlinearly dependent on the reduction in initial ice crystal numbers. A reduction in the initial ice crystal number of $80 \%$ leads to a decrease in contrail cirrus radiative forcing by $50 \%$, whereas a twofold reduction leads to a decrease in radiative forcing by approximately $20 \%$. Only a few contrail cirrus outbreaks explain a large percentage of the climate impact. The contrail cirrus climate impact can be effectively mitigated by reducing initial ice crystal concentrations in such outbreak situations. Our results are important for assessments dealing with mitigating the climate impact of aviation and discussions about the use of alternative fuels or lean combustion in aviation.

npj Climate and Atmospheric Science (2018)1:37; doi:10.1038/s41612-018-0046-4

\section{INTRODUCTION}

Aviation modifies cloudiness directly due to the formation of contrails and indirectly due to aerosol effects on clouds increasing cloudiness and changing optical properties of cirrus. ${ }^{1-3}$ Contrails reflect solar short-wave radiation and trap outgoing long-wave radiation. ${ }^{4}$ As opposed to low clouds, thin high clouds, such as contrails, have on average a larger impact on the outgoing longwave than on the solar short-wave radiation, exerting on average a warming at the top of the atmosphere. ${ }^{4-6}$ Aviation contributes approximately $5 \%$ to the anthropogenic climate forcing ${ }^{7}$ with contrail cirrus being the largest known radiative forcing component ${ }^{8,9}$ and the indirect aerosol effect on clouds constituting a significant but highly uncertain contribution. ${ }^{10-13}$ One possible option, mitigating the aviation climate impact, is reducing aviation soot number emissions by e.g. using alternative fuels ${ }^{14,15}$ or reducing the aromatic content of fuel. Estimating the effectiveness of this option is comparatively straightforward since, besides the uncertain indirect aerosol effect, only one climate forcing component, contrail cirrus, is significantly affected. The impact of this mitigation option on climate is, therefore, not dependent on a possibly fine balance between compensating climate forcings.

Contrails form in the wake of aircraft if water saturation is reached or surpassed during the mixing of exhaust and environmental air. ${ }^{16}$ The vast majority of contrail ice crystals form when emitted soot particles and ambient aerosol particles entrained into the plume activate to droplets in water supersaturated conditions and freeze subsequently by homogeneous nucleation. ${ }^{17-19}$ Some plume aerosol particles may act as ice nuclei forming ice crystals in ice supersaturated conditions but their number is too small to have a significant impact on initial contrail properties due to the large plume cooling rates. Contrails develop and persist in ice supersaturated areas at humidities lower than the threshold for in-situ cirrus formation ${ }^{20}$ increasing upper tropospheric cloudiness. ${ }^{1}$ In large-scale ice supersaturated areas contrail lifetimes can be long, exceeding occasionally $17 \mathrm{~h}$, and add significantly to cloud coverage..$^{21-24}$ The radiative impact of contrail cirrus is mainly dependent on their coverage and optical depth. ${ }^{5}$

A 50:50 mix of a HEFA biofuel and regular Jet $A$ fuel was found to decrease soot number emissions from airplanes by $50-70 \%{ }^{14}$ Decreasing the fuel aromatics content, using biofuel together with liquid natural gas or liquid hydrogen, ${ }^{25}$ or switching to lean combustion $^{26}$ is expected to lead to even larger reductions. Reducing engine soot number emissions by up to one order of magnitude from current levels $\left(\sim 1^{*} 10^{15} \text { per kg fuel }\right)^{27}$ leads to approximately the same reduction in the number of nucleated ice crystals as long as atmospheric temperatures are at least $\sim 2 \mathrm{~K}$ below the threshold temperature for contrail formation. ${ }^{18,19}$ Reducing soot emissions even further, would require considering additionally the impact of volatile plume particles on ice nucleation. ${ }^{17,18}$ The temperature threshold is usually well exceeded at cruise levels in the extra-tropics, where most of the air traffic is located. During the contrail's vortex phase ice crystal numbers are sublimated within the descending vortex pair. ${ }^{28-30}$ The loss is stronger for many small ice crystals, partly compensating for ice crystal number differences. Later during the contrail's life cycle when ice crystal sedimentation becomes significant, the difference in ice crystal numbers increases again since fewer ice crystals lead to faster growth and increased sedimentation into ice-subsaturated lower levels. ${ }^{24}$

${ }^{1}$ Deutsches Zentrum für Luft- und Raumfahrt (DLR), Institut für Physik der Atmosphäre, Oberpfaffenhofen, Germany

Correspondence: Ulrike Burkhardt (Ulrike.burkhardt@dlr.de)

Received: 22 September 2017 Revised: 24 August 2018 Accepted: 28 August 2018

Published online: 19 October 2018 
The changes in the microphysical properties of contrail cirrus caused by reduced soot number emissions can be expected to modify their climate impact in a similar way than a reduction in aerosol loading of the atmosphere would modify that of liquid clouds: A smaller number of larger contrail ice crystals acts to decrease contrail optical depth (cloud albedo effect) (, $^{9,31}$ and shortens contrail lifetime (lifetime effect). ${ }^{9,32}$ We use the CCMod contrail cirrus parameterization ${ }^{33}$ within the ECHAM5 $5^{34,35}$ climate model in order to study the impact of reduced soot number emissions on optical properties, lifetimes and radiative forcing and estimate the mitigation potential when reducing soot number emissions in specific meteorological situations. We study exemplarily a reduction in initial ice crystal numbers by $80 \%$, a reduction that may lie in between the reductions expected from using a 50:50 blend of biofuel and regular Jet A fuel and from lean combustion. ${ }^{26}$ Additionally we look at the impact of assuming a lower $(50 \%)$ or higher (90\%) reduction in initial ice crystal numbers.

\section{RESULTS}

Change in contrail cirrus properties

Contrail cirrus ice water content at $240 \mathrm{hPa}$, a pressure level close to the main flight level, is significantly decreased and ice crystal sizes are increased (Fig. 1a,b) when prescribing decreased initial ice crystal numbers. The changes in the two fields and in particular their large regional variability can be explained when considering the contrail cirrus life cycle and the large impact that a reduction in soot number emissions has on the life cycle. At the beginning of the life cycle ice water mass is limited by the water vapor available for deposition. ${ }^{33}$ For reduced initial ice crystal numbers this water vapor is deposited on fewer ice crystals leading to strongly increased ice crystal sizes. Total ice water mass within the contrail cirrus volume, on the other hand, is not significantly changed; in fact, for reduced initial ice crystal numbers, it is slightly increased in the first few hours $(<\sim 4 \mathrm{~h})$ due to the larger increase in the contrail cross sectional area, or volume, caused by increased sedimentation rates. ${ }^{24}$ In the later part of the life cycle the increase in the contrail cirrus ice water content is limited by the low ice crystal number concentration and not by the water vapor available for deposition within the contrail cirrus volume. ${ }^{33}$ During that part of the life cycle total ice water mass is reduced due to a reduction in initial ice crystal number whereas ice crystal sizes are hardly changed. ${ }^{33}$ This means that the impact of changed initial ice crystal numbers on the total contrail cirrus ice water mass increases with contrail lifetime while the impact on ice crystal sizes decreases. On average the total ice water mass of contrail cirrus is considerably higher for the 'present-day soot number scenario' (Fig. 1a) owing to the fact that the lower total ice water mass early in the contrail cirrus' life cycle is more than compensated for in the later part of the life cycle.

The absolute change in contrail cirrus ice water content is mainly dependent on latitude, with maximum values in the tropics. The relative change in ice water content (Fig. 1a) is smallest in the main air traffic areas, where contrail cirrus largely consist of young contrails and, as explained above, deposition is limited by availability of water vapor. In those main air traffic areas the relative change in ice crystal sizes (Fig.1b) is largest. In areas where air traffic is low and contrail cirrus mainly consist of aged contrail cirrus with low ice crystal number concentrations, ${ }^{36}$ the relative change in ice water content is large since deposition is limited by ice crystal number concentrations. In those areas the relative change in ice crystal sizes is small.

The mean contrail cirrus optical depth generally decreases with a reduction in ice crystal numbers. This decrease is caused by the unchanged ice water content at increased ice crystal sizes ${ }^{37}$ at the beginning of the life cycle and later by the decrease in ice water content at unchanged ice crystal sizes. These changes are very pronounced in the main air traffic areas, such as over Northern America or Europe (Fig. 1e). Over Europe, very low solar optical depths $(\leq 0.05)$ are double as common and the probability of an optical depth $>0.1$ is reduced by more than $80 \%$. Since a solar optical depth of 0.05 marks approximately the threshold for the detection of contrails in satellite images, ${ }^{38}$ this means that contrail cirrus coverage as obtained from satellite observations would be largely reduced.

\section{Contrail cirrus cover and radiative forcing}

Due to the $80 \%$ decrease in the initial ice crystal number ('low soot number scenario'), contrail cirrus coverage is decreasing significantly. For present-day soot number emissions, the globally averaged coverage due to contrail cirrus with a solar optical depth $>0.05$ is $0.47 \%{ }^{36}$; in the 'low soot number scenario' this globally averaged coverage amounts to $0.2 \%$, a value that is $41 \%$ lower than in the 'present-day soot number scenario'. Maxima in coverage are located over Europe and the USA and reach in the'low soot number scenario' $12 \%$ and $8 \%$, respectively. This is an absolute reduction of contrail cirrus coverage over Europe and the USA of around 1\% (Fig. 1c). Considering contrail cirrus with a solar optical depth $>0.02$, global mean contrail cirrus coverage for present-day soot number emissions is $0.6 \%$. For the 'low soot number scenario' the respective value is $0.37 \%$, which is, relative to the 'present-day soot number scenario' a reduction by $38 \%$. The reduction of contrail cirrus cover is largest in the main air traffic areas of the northern extratropics. Reductions are locally small over central Europe and the USA south of the Great Lakes, both areas with high air traffic and a minimum relative decrease in contrail cirrus optical depth. The decrease in the cover of contrail cirrus with solar optical depth $>0.05$ is mainly due to the drop in contrail cirrus optical depth and only to a lesser degree due to a decrease in the overall contrail cirrus coverage.

Accordingly, when reducing the initial ice crystal number by $80 \%$, global contrail cirrus net radiative forcing is decreased by $50 \%$ (Fig.1f), from $35 \mathrm{mWm}^{-2}$ (for the 'present-day soot number scenario') to $17 \mathrm{mWm}^{-2}$ (for the 'low soot number scenario'). The net radiative forcing for the 'low soot number scenario' results from a long-wave radiative forcing of $34 \mathrm{mWm}^{-2}$ and a shortwave forcing of $-16 \mathrm{mWm}^{-2}$ compensating the long-wave forcing by $47 \%$. The compensation may be slightly decreased relative to the 'present-day soot number scenario' where long-wave forcing amounted to $72 \mathrm{mWm}^{-2}$ and compensation due to SW forcing was $51 \%$. In the main air traffic areas of central Europe and the northern USA the reduction in contrail cirrus radiative forcing exceeds in large areas $100 \mathrm{mWm}^{-2}$ (Fig. 1d), so that the area, where radiative forcing exceeds $500 \mathrm{mWm}^{-2}$, is significantly reduced for reduced initial ice crystal numbers. Relative to its mean the percentage change in radiative forcing lies below $40 \%$ in the main air traffic areas of the USA and Europe and in most other areas at or above $60 \%$.

The reduction in net contrail cirrus radiative forcing (short-wave plus long-wave radiative forcing) is nonlinearly dependent on the reduction in initial ice crystal number (Fig. 1f). Reducing the initial ice crystal number by $90 \%$ (to 0.1 of the present-day values), global contrail cirrus radiative forcing is reduced by $69 \%$. Reducing initial ice crystal number by only $50 \%$ (to 0.5 of the present-day values), a value that may be obtained when using a 50:50 blend of an alternative fuel and Jet $A$ fuel, leads to a reduction in radiative forcing of about $21 \%$.

\section{Contrail cirrus life cycle}

In order to study the processes responsible for the reduction in global contrail cirrus cover and radiative forcing, we conduct experiments simulating single contrail cirrus clusters forming within $1 \mathrm{~h}$ in a limited domain for present-day and low soot 
[\%]
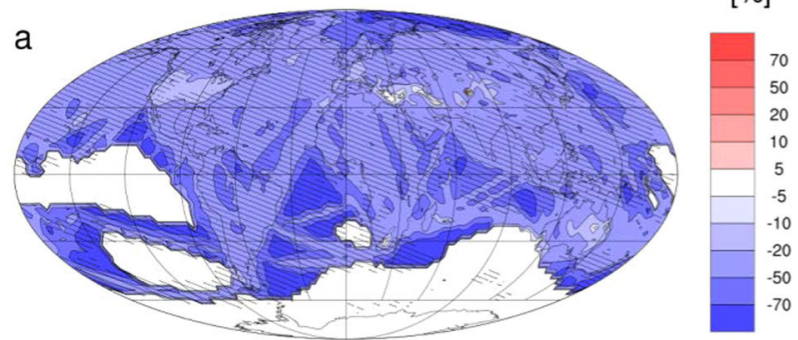

[\%]
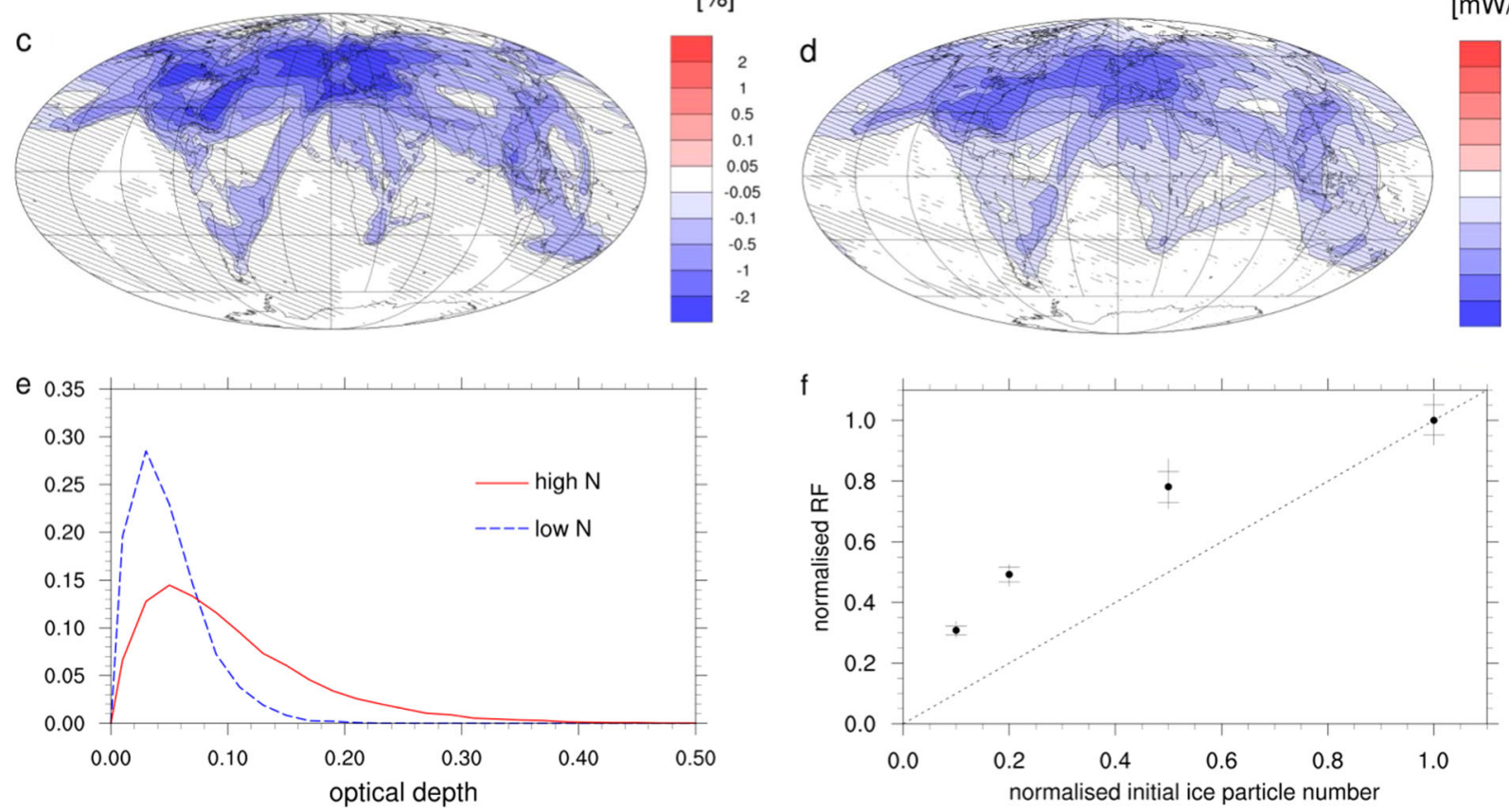

Fig. 1 Impact of a reduction in initial ice crystal number, as may be caused by reduced soot emissions, on global contrail cirrus properties. Relative change in contrail cirrus a in-cloud ice water content and $\mathbf{b}$ ice crystal volume radius at $240 \mathrm{hPa}$ and absolute change in (c) total cover of contrail cirrus with an optical depth $>0.05$ and $\mathbf{d}$ contrail cirrus net radiative forcing due to a $80 \%$ reduced initial ice crystal number (low minus present-day initial ice crystal number). Hatched areas indicate locations in which the difference is statistically significant at a $95 \%$ confidence level. e Frequency of contrail cirrus optical depth over Europe at $240 \mathrm{hPa}$ when prescribing present-day (red), or low (blue) initial ice crystal number concentration. $f$ Global net radiative forcing (RF), given as a fraction of the radiative forcing for the 'present-day soot number scenario', as a function of the initial ice particle number concentration of contrails, given as a fraction of the initial ice crystal number concentration for the 'present-day soot number scenario'. Initial ice crystal numbers were reduced to $0.5,0.2$, and 0.1 of the present-day values. Vertical bars (horizontal lines) indicate the full range ( $90 \%$ of the range) of values obtained when calculating the reduction based on yearly means. The dashed line, indicating linearity, serves for comparison

number emissions and analyze their evolution. We studied life cycles of contrail cirrus clusters originating in the extratropics, over the eastern USA $\left(35^{\circ} \mathrm{N}-45^{\circ} \mathrm{N}, 85^{\circ} \mathrm{W}-100^{\circ} \mathrm{W}\right)$, and in the tropics, over the central part of Southeast Asia $\left(4^{\circ} \mathrm{N}-14^{\circ} \mathrm{N}\right.$ and $\left.95^{\circ} \mathrm{E}-110^{\circ} \mathrm{E}\right)$. The mean maximum total extinction, a measure for the shortwave radiative impact of the contrail cirrus clusters, is in the tropics lower than in the extratropics by about a factor of 8 (Fig. 2). This results mainly from the lower contrail coverage in the tropics ${ }^{36}$ caused by a combination of lower air traffic density and a lower contrail formation probability. On average, the lifetime of contrail cirrus clusters is approximately $4 \mathrm{~h}$ shorter in the tropics than in the extratropics, resulting mainly from the enhanced ice crystal growth and sedimentation rates in the tropics due to the higher specific humidity. Tropical contrail lifetimes are likely to be even smaller when considering the dependency of contrail ice nucleation on the atmospheric state. ${ }^{17}$

For reduced initial ice crystal numbers, mean maximum total extinction is in both areas significantly reduced by about a factor of 2 and is reached on average 1-2 h earlier. The decline of contrail cirrus optical depth and total extinction is mainly caused by the limitation of water vapor deposition due to low ice crystal number concentrations ${ }^{24}$ and therefore occurs earlier in contrail cirrus clusters with low initial ice crystal numbers. The lifetime of the contrail cirrus clusters with reduced initial ice crystal numbers is on average significantly reduced by $4-5 \mathrm{~h}$ relative to the lifetime connected with present-day initial ice crystal numbers. Integrated over the lifetime, total extinction is decreased on average by about $65 \%$ both for the tropical and the extratropical area.

This reduction is larger than the reduction found in the global simulations with prescribed global air traffic (Fig. 1). When prescribing global air traffic at each time step, air traffic occurring in aged contrail cirrus tends to reinvigorate older contrail cirrus by the addition of new ice crystals ${ }^{36}$ increasing the optical depth of older contrail cirrus. This reinvigoration leads in the global simulations within the high air traffic density areas, over Europe and Northern America, to very high ice crystal number concentrations so that contrail cirrus radiative forcing is decreased by less than $50 \%$ (Fig.1d) when reducing initial ice crystal numbers by $80 \%$. Over the Northern Atlantic this decrease amounts to around $70 \%$. 

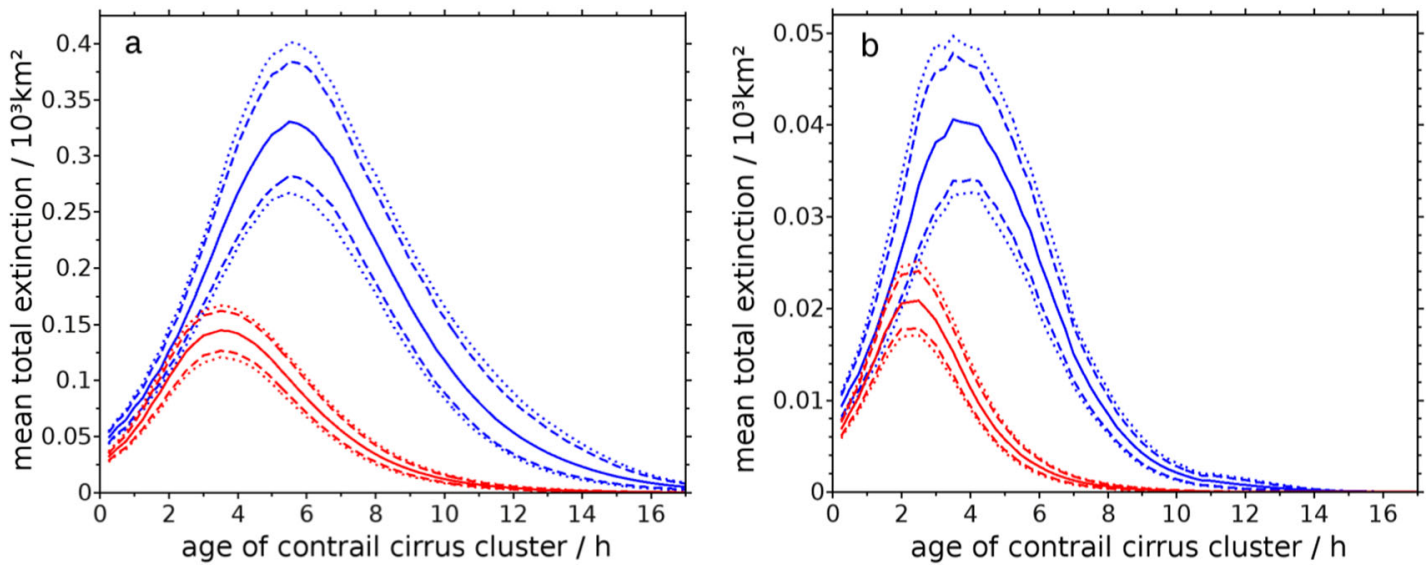

Fig. 2 Life cycle of contrail cirrus clusters. Average life cycle of the short-wave radiative impact (total extinction) of a contrail cirrus cluster originating (left) over the eastern USA $\left(35^{\circ} \mathrm{N}-45^{\circ} \mathrm{N}, 85^{\circ} \mathrm{W}-100^{\circ} \mathrm{W}\right)$ and (right) over the central part of Southeast Asia $\left(4^{\circ} \mathrm{N}-14^{\circ} \mathrm{N}\right.$ und $\left.95^{\circ} \mathrm{E}-110^{\circ} \mathrm{E}\right)$ when prescribing present-day (blue) and low (red) initial ice crystal number concentrations associated with the 'present-day soot number scenario'and 'low soot number scenario'. Dashed (dotted) lines indicate the $95 \%$ (99\%) confidence interval calculated using the bootstrapping method $^{35}$
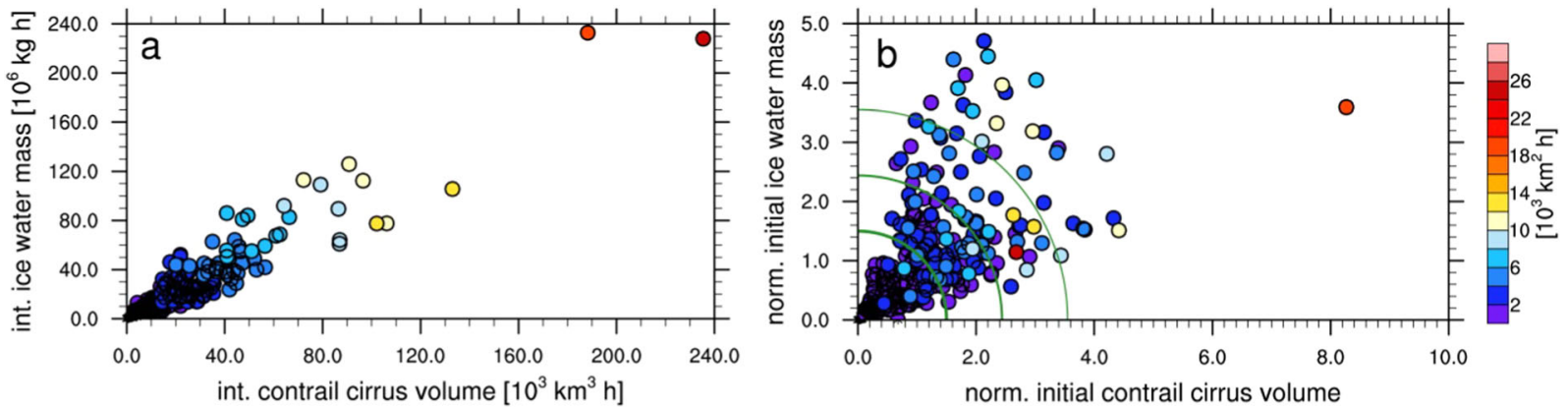

Fig. 3 Inferring the short-wave climate impact of contrail cirrus clusters from the integrated or initial contrail cirrus ice water mass and volume. Short-wave climate impact (total extinction) of contrail cirrus clusters integrated over the whole life cycle (colored points) as a function of $\mathbf{a}$ the contrail cirrus volume and ice water mass each integrated over the life cycle of the contrail cirrus cluster and $\mathbf{b}$ the initially formed contrail volume and ice water mass. In $\mathbf{b}$ the initially formed contrail volume and ice water mass were each normalized by their respective standard deviations. Each colored point represents a contrail cirrus cluster with the color indicating the short-wave radiative impact integrated over the cluster's lifetime. The green circles in b contain the 50,75 and $90 \%$ of contrail cirrus clusters with the smallest initial contrail volume and ice water mass

\section{High impact contrail cirrus outbreaks}

The life cycle of contrail cirrus clusters and the impact of a reduction in initial ice crystal number are in the extratropics largely controlled by the synoptic situation. ${ }^{24}$ Contrails that form within large-scale ice supersaturated areas in the upper troposphere often persist for a long time spreading considerably within those areas. In this synoptic situation when the upper troposphere supports large scale contrail cirrus outbreaks, a reduction in initial ice crystal numbers has a large impact. On other days the impact is low, making any mitigation effort ineffective. From the life cycles of the contrail cirrus clusters originating over the Eastern USA, we estimate that the $10 \%$ largest contrail cirrus clusters, as measured by the effect on short-wave radiation (total extinction), are responsible for $42 \%$ of the short-wave radiative impact of all contrail cirrus clusters. The largest $25 \%$ of clusters contribute $68 \%$ of the radiative impact. Good predictors for the short-wave radiative impact are contrail cirrus volume and total ice water mass of the contrail cirrus cluster integrated over the life cycle of the cluster (Fig. 3a). Whenever the integrated contrail cirrus volume is large, the integrated ice water mass and the total extinction are very likely to be large as well.

We estimate the reduction in the contrail cirrus climate impact when reducing initial ice crystal numbers in only those synoptic situations when cover and ice water mass integrated over the contrail cirrus life cycle are large. Based on previous results, ${ }^{24}$ we can assume that to a good approximation the reduction in initial ice crystal number causes a reduction in the contrail cirrus radiative impact with the relative size of the reduction independent of the synoptic situation. With an $80 \%$ reduction in initial ice crystal number contrail cirrus radiative forcing is reduced by about $50 \%$ (Fig. 1f) in the simulations for global air traffic. Reducing initial ice crystal numbers only in those 10 and $25 \%$ of synoptic situations in which contrail cirrus clusters obtain the largest integrated contrail cirrus cover and ice water mass, we estimate that the contrail cirrus radiative impact can be reduced by nearly $21 \%$ and nearly $35 \%$, respectively. If we were able to forecast those situations that support highly climate relevant contrail cirrus outbreaks and if we were able to reduce soot emissions dependent on the synoptic situation, we could mitigate a significant portion of the aviation climate impact.

From numerical weather forecasts the initial contrail cirrus volume and ice water mass can be estimated from the spatial extent and the magnitude of ice supersaturation. We use the initial contrail cirrus volume and ice water mass as predictors for the short-wave radiative impact of the contrail cirrus clusters (Fig. $3 b)$. Situations with large initial ice water mass exist, for which integrated total extinction remains small (blue points far away from the axis origin) and the highest total extinction can be found for a situation that is not connected with a particularly large initial volume or ice water mass (red points within the third green circle). 
Nevertheless, we find that both predictors together have considerable skill in forecasting contrail cirrus clusters with a high radiative impact. The 10 and $25 \%$ of events with the highest predicted contrail cirrus climate impact (high initial contrail volume and ice water content) amount to 27 and $57 \%$ of the overall short-wave radiative impact, respectively. Reducing the initial ice crystal number by $80 \%$ for those top 10 and $25 \%$ events only, would lead to a reduction of contrail cirrus radiative impact of close to $15 \%$ and close to $30 \%$, respectively.

\section{DISCUSSION}

We report an estimate for the change in the aviation climate impact when reducing soot number emissions by e.g. switching to alternative fuels or reducing the aromatics content of fuel. This change results predominantly from changes in contrail cirrus properties due to a reduction in soot number emissions. Different mitigation methods for reducing soot number emissions have varying degrees of effectiveness. We analyze exemplarily a reduction in initial ice crystal number of $80 \%$ and vary this reduction, calculating radiative forcing for a 50 and $90 \%$ reduction. When reducing engine soot number emissions, initial ice crystal numbers are reduced, leading to changes in the properties, the life cycle and radiative forcing of contrail cirrus. Contrail cirrus ice water content is strongly reduced and ice crystal sizes increased leading to a large reduction in contrail cirrus optical depth (cloud albedo effect) ${ }^{9,31}$ and doubling the fraction of contrail cirrus that cannot be detected by remote sensing methods. The reduction in the initial ice crystal numbers causes changes in the microphysical process rates, leading to a reduction in the contrail cirrus lifetimes (life time effect) ${ }^{9,32}$ and in contrail cirrus coverage. Both, a shorter lifetime (4-5 $\mathrm{h}$ on average) and lower optical depth of the contrail cirrus clusters are contributing to the reduction in contrail cirrus radiative forcing. Global contrail cirrus radiative forcing is nonlinearly dependent on the initial ice crystal number and can be reduced by approximately $50 \%$ when decreasing the initial ice crystal number by about $80 \%$. Since the indirect aerosol effect is uncertain, this reduction in contrail cirrus radiative forcing translates into a reduction of the aviation radiative forcing since decreased soot number emissions affect predominantly the climate impact of contrail cirrus.

Only a few large-scale contrail cirrus outbreaks were found to be responsible for a large part of the radiative forcing. Mitigating the climate impact of contrail cirrus by reducing soot number emissions only in those synoptic situations that support large scale contrail outbreaks, the climate impact can be reduced significantly. Inferring those situations from a numerical weather forecast (assuming the forecast to be perfect) and reducing soot number emissions by $80 \%$ in those $25 \%$ of the situations, for which a high contrail cirrus climate impact can be expected, could lead to a reduction in time averaged contrail cirrus radiative forcing of about $30 \%$.

In order to calculate the soot number emission reduction that is necessary to achieve a certain reduction in the initial ice crystal number, ice nucleation and ice crystal loss within the vortex phase, both dependent on the synoptic situation, need to be considered. Whereas in the extratropics a reduction in soot number emissions at cruise levels leads to a good approximation to an equal reduction in contrail ice nucleation as long as soot emissions are above $10^{14}$ per kg-fuel, the subsequent vortex phase tends to counteract this reduction. A reduction in nucleated ice crystal numbers by one order of magnitude down from $10^{15} \mathrm{~kg}^{-1}$ may lead on average to an $80 \%$ reduction in ice crystal number after the vortex phase. ${ }^{29}$ Since the vortex phase loss tends to be smaller in the very climate relevant large-scale highly ice supersaturated states, the required reduction in soot number emissions lies below one order of magnitude, likely at around $90 \%$.
A change in contrail cirrus radiative forcing cannot be easily translated into a change in the contrail cirrus climate impact, when measured in terms of surface temperatures, since contrail cirrus lifetimes and the impact of radiative forcing on surface temperatures need to be considered. ${ }^{41,42}$ Finally, if aviation soot number emission reductions are to be introduced by a switch to alternative fuels, an evaluation of the climate impact of replacing conventional kerosene by alternative fuel needs to consider the whole fuel life cycle including the production and distribution of alternative fuels.

\section{METHODS}

The contrail-cirrus module, CCMod, introduces a new cloud class 'contrail cirrus' in the global climate model ECHAM5 ${ }^{34,35}$ ECHAM5 has a diagnostic relative-humidity based cloud-cover scheme ${ }^{43}$ and a 2-moment microphysical scheme, ${ }^{44}$ which was modified regarding the ice nucleation below $-40^{\circ} \mathrm{C}$ to be consistent with the original fractional diagnostic cloud coverage. $^{33}$ ECHAM5-CCMod ${ }^{33}$ is an advanced version of the ECHAM4CCMod mode ${ }^{45}$ including a 2-moment microphysical scheme for contrails.

CCMod is based on a prognostic treatment of contrail cirrus fractional coverage, volume, length, ice crystal number concentration and ice water mass mixing ratio. The processes controlling contrail cirrus properties, namely contrail formation below a threshold temperature, ${ }^{16}$ volume growth due to turbulent diffusion and sedimentation ${ }^{33}$ and tilting due to vertical wind shear, ${ }^{45}$ are parametrized physically consistent with the parametrization of natural clouds. Water vapor deposition within contrails is limited by contrail cirrus volume and ice crystal number concentration, the latter limiting deposition at low ice crystal number concentrations ${ }^{33}$ due to long diffusional growth time scales. Microphysical processes, except nucleation, are treated in the same way as for natural clouds with a size dependency of microphysical processes, such as sedimentation, captured by the microphysical two-moment scheme. Of the flight distance, only the fraction of flights taking place in ice supersaturated areas results in persistent contrails. Contrails are advected by the wind field and are limited by the ice-supersaturated fraction of a grid box. Ice supersaturated areas are inferred from the assumptions of subgrid-scale variability given by the cloud scheme. ${ }^{46}$ The model's water budget is changed to accommodate for the new cloud class, ${ }^{45}$ enabling the simulation of the competition for available water vapor between natural and contrail cirrus.

The model simulates the life cycle of contrail cirrus, starting from the state after the vortex phase. It reproduces many properties found in $\mathrm{LES}^{28,30,40}$ such as the dilution and decreasing ice crystal number concentration in the dispersion phase of the young contrail, the initial increase in contrail cirrus ice water content and optical depth and the subsequent decline at a time when ice crystal number concentrations are severely diminished. ${ }^{33}$ CCMod has been evaluated using satellite and in situ measurements of ice supersaturation ${ }^{33,46,47}$ and of young contrail properties and cover. ${ }^{29,36,45}$

We conduct global simulations using the global air traffic inventory AERO2 $\mathrm{k}^{48}$ for the year 2002. Integrations over 5 and 15 years with the ECHAM5-CCMod climate model (using a time step of $15 \mathrm{~min}$, a horizontal resolution of T42 and 41 vertical levels) have been carried out, prescribing constant initial ice crystal number concentrations that are representative of different soot number emissions, in order to estimate contrail-cirrus coverage and radiative forcing. For the 'present-day soot number scenario', representing current soot number emissions, we prescribe a constant initial ice crystal number concentration of $150 \mathrm{~cm}^{-3}$, an average value derived from measurements of contrails with an age of a few minutes. ${ }^{49}$ This ice crystal number concentration, at $7.5 \mathrm{~min}$ after contrail formation, corresponds to an apparent ice crystal number emission index of 4.5 to $5.5 \cdot 10^{14}$ per kg fuel when disregarding the decrease of ice crystal numbers within the vortex phase. We call this initialization the 'present-day soot number emissions' scenario. We approximate the effect of reduced soot number emissions by a fixed decrease in the initial ice crystal number ('low soot number emissions' scenario). Since different measures for decreasing soot emissions, such as a decrease in the aromatic content of fuel, mixing regular Jet-A fuel with biofuel, ${ }^{14}$ using multi-fuel approaches ${ }^{25}$ or moving to lean combustion, ${ }^{26}$ result in the reduction of soot number emissions by differing amounts, we reduce initial ice crystal concentration by 50,80 and $90 \%$ and calculate the reduction in radiative forcing.

Stratosphere-adjusted radiative forcing has been calculated as a difference between two calls of the radiation scheme at each time step in a model run, ${ }^{50}$ allowing the online calculation of radiative forcing due to 
contrail cirrus. For the radiation calculations, natural clouds and contrail cirrus have been randomly overlapped in the vertical at each model time step, except when clouds existed in neighboring model levels, in which case they were assumed to overlap maximally (maximum random overlap). This allows natural clouds and contrail cirrus to overlap each other in the vertical.

We conduct life cycle experiments in order to analyze the processes leading to the change in radiative forcing when initial ice crystal numbers are reduced by $80 \%$. In those experiments we prescribe air traffic within two specified domains $\left(35^{\circ} \mathrm{N}-45^{\circ} \mathrm{N}, 85^{\circ} \mathrm{W}-100^{\circ} \mathrm{W}\right.$ and $4^{\circ} \mathrm{N}-14^{\circ} \mathrm{N}$ und $95^{\circ}$ $\mathrm{E}-110^{\circ} \mathrm{E}$ ) at $220 \mathrm{hPa}$ for $1 \mathrm{~h}$ only, analyzing the evolution of the contrail cirrus cluster after contrail formation. By restricting air traffic to those two locations and a short time period, we are able to analyze the life cycle of a cluster of contrail cirrus (all of the same age) developing in a specific synoptic situation. The area over the eastern USA is representative of the extratropical main air traffic area. In this area contrail cirrus outbreaks are observed frequently. We analyze total extinction, ${ }^{24,40}$ a measure for the short-wave effect of contrail cirrus. Total extinction gives the fraction of incident short-wave radiation that is scattered or reflected by the contrail cirrus ice crystals and is dependent on contrail cirrus optical depth and total cover. It is therefore independent of the time of day and variations in the background meteorology and surface conditions.

Statistical significances have been calculated in Fig. 1a-d using the twosided Student's $t$ test taking into account that monthly mean values may be serially correlated and calculating the number of independent time steps. ${ }^{39}$ The original simulations were 15 years (present-day soot number scenario) and 5 years (reduced soot number scenarios) long. The error bar in Fig. If represents the variability when calculating the reduction in radiative forcing from the yearly mean values. We assume independence of the two simulations and calculate 75 realizations of the reduction of radiative forcing indicating absolute maxima and minima and the $90 \%$ interval of the distribution. The confidence interval in Fig. 2 was calculated using the bootstrapping method ${ }^{39}$ drawing from a pool of 261 (268) simulated contrail cirrus cluster life cycles for present-day (low) soot emissions and calculating the 95 and $99 \%$ confidence interval.

\section{DATA AVAILABILITY}

Data are available at https://www.pa.op.dlr.de/opendata/20171010UB.

\section{ACKNOWLEDGEMENTS}

We thank Mattia Righi for comments on the paper. L.B. was supported by the Bundesministerium f ur Wirtschaft und Technologie (BMWi) as a result of a decision of the Deutsche Bundestag within the project ATMOSFAIR (20Y0904D). A.B. has carried out research within the DLR project 'Emission and CLimate Impact of alternative Fuels (ECLIF)'. Computational resources were made available by the German Climate Computing Centre (DKRZ) through support from the German Federal Ministry of Education and Research

\section{AUTHOR CONTRIBUTIONS}

L.B. performed and analyzed simulations on the global climate impact of air traffic. A. B. performed and analyzed simulations of contrail cirrus clusters. U.B. lead research, performed mitigation statistics and wrote the paper.

\section{ADDITIONAL INFORMATION}

Competing interests: The authors declare no competing interests.

Publisher's note: Springer Nature remains neutral with regard to jurisdictional claims in published maps and institutional affiliations.

\section{REFERENCES}

1. Seinfeld, J. H. Clouds Contrails and climate. Nature 391, 837-838 (1998).

2. Boucher, O. Air traffic may increase cirrus cloudiness. Nature 397, 30-31 (1999).

3. Tesche, M., Achtert, P., Glantz, P. \& Noone, K. J. Aviation effects on already existing cirrus clouds. Nat. Commun. 7, 12016 (2016).

4. Minnis, P. Encyclopedia of Atmospheric Sciences. 509-12520 (Academic Press, London, 2003).

5. Meerkötter, R. et al. Radiative forcing by contrails. Ann. Geophys. 17, 1080-1094 (1999).
6. Myhre, G. et al. Intercomparison of radiative forcing calculations of stratospheric water vapor and contrails. Met. Z., 18, 585-596 (2009).

7. Lee, D. S. et al. Aviation and globalclimate change in the 21 st century. Atmos. Environ. 43, 3520-3537 (2009).

8. Burkhardt, U. \& Kärcher, B. Global radiative forcing from contrail cirrus. Nat. Change 1, 54-58 (2011).

9. IPCC climate change 2013: the physical science basis. In: contribution of Working Group I to the Fifth Assessment Report of the Intergovernmental Panel on Climate Change. Cambridge University Press, Cambridge, United Kingdom and New York, NY, USA.

10. Gettelman, A. \& Chen, C. The climate impact of aviation aerosols. Geophys. Res. Lett. 40, 2785-2789 (2013).

11. Righi, M., Hendricks, J. \& Sausen, R. The global impact of the transport sectors on atmospheric aerosol: simulations for year 2000 emissions. Atmos. Chem. Phys. 13, 9939-9970 (2013).

12. Kapadia, Z. Z. et al. Impacts of aviation fuel sulfur content on climate and human health. Atmos. Chem. Phys. 16, 10521-10541 (2016).

13. Zhou, C. \& Penner, J. E. Aircraft soot indirect effect on large-scale cirrus clouds: is the indirect forcing by aircraft soot positive or negative? J. Geophys. Res. Atmos. 119, 11303-11320 (2014).

14. Moore, R. H. et al. Biofuel blending reduces particle emissions from aircraft engines at cruise conditions. Nature 543, 411-415 (2017).

15. Kärcher, B. The importance of contrail ice formation for mitigating the climate impact of aviation. J. Geophys. Res. Atmos. 121, 3497-3505 (2016).

16. Schumann, U. On conditions for contrail formation from aircraft exhausts. Meteorol. Z. 5, 4-24 (1996).

17. Kärcher, B., Burkhardt, U., Bier, A., Bock, L. \& Ford, I. J. The microphysical pathway to contrail formation. J. Geophys. Res. Atmos. 120, 7893-7927 (2015).

18. Kärcher, B. \& Yu Role, F. Of aircraft soot emissions in contrail formation. Geophys. Res. Lett. 36, L01804 (2009).

19. Wong, H.-W. \& Miake-Lye, R. C. Parametric studies of contrail ice particle formation in jet regime using microphysical parcel modeling. Atmos. Chem. Phys. 10, 3261-3272 (2010).

20. Koop, T., Luo, B. P., Tsias, A. \& Peter, T. Water activity as the determinant for homogeneous ice nucleation in aqueous solutions. Nature 406, 611-614 (2000).

21. Minnis, P. et al. Transformation of contrails into cirrus during SUCCESS. Geophys. Res. Lett. 25, 1157-1160 (1998).

22. Haywood, J. M. et al. A case study of the radiative forcing of persistent contrails evolving into contrail-induced cirrus. J. Geophys. Res. 114, D24201 (2009).

23. Vazquez-Navarro, M., Mannstein, H. \& Kox, S. Contrail life cycle and properties from 1 year of MSG/SEVIRI rapid-scan images. Atmos. Chem. Phys. 15, 8739-8749 (2015).

24. Bier, A., U. Burkhardt and L. Bock Synoptic control of contrail cirrus life cycles and their modification due to reduced soot number emissions. accepted by J. Geophys. Res. Atmospheres 122, 11, 584-11, 603 (2017).

25. Grewe, V. et al. Assessing the climate impact of the AHEAD multi-fuel blended wing body. Met. Z. 26, 711-725 (2017).

26. Foust, M., Thomsen, D., Stickles, R., Cooper, C., and Dodds, W. "Development of the GE aviation low emissions taps combustor for next generation aircraft engines," AIAA Paper, October (2011).

27. Schumann, U., Jeßberger, P. \& Voigt, C. Contrail ice particles in aircraft wakes and their climatic importance. Geophys. Res. Lett. 40, 2867-2872 (2013).

28. Paoli, R. \& Shariff, K. Contrail modeling and simulation. Annu. Rev. Fluid. Mech. 48, 393-427 (2016).

29. Unterstrasser, S. Properties of young contrails - a parameterization based on large-eddy simulations. Atmos. Chem. Phys. 16, 2059-2082 (2016).

30. Lewellen, D. C. Persistent contrails and contrail cirrus. Part II: full lifetime behavior. J. Atmos. Sci. 71, 4420-4438 (2014).

31. Twomey, S. Pollution and the planetary albedo. Atmos. Environ. 8, 1251-1256 (1974).

32. Albrecht, B. A. Aerosols, cloud microphysics, and fractional cloudiness. Science 245, 1227-1230 (1989).

33. Bock, L. \& Burkhardt, U. The temporal evolution of a long-lived contrail cirrus cluster: simulations with a global climate model. J. Geophys. Res. Atmos. 121, 3548-3565 (2016).

34. Roeckner, E. et al. The atmospheric general circulation model ECHAM5. Part 1: model description, Max-Planck-Inst. Report 349, 127 pp., Hamburg, Germany (2003).

35. Roeckner, E. et al. Sensitivity of simulated climate to horizontal and vertical resolution in the ECHAM5 atmosphere model. J. Clim. 19, 3771-3791 (2006).

36. Bock, L. \& Burkhardt, U. Reassessing properties and radiative forcing of contrail cirrus using a globale climate model. J. Geophys. Res. Atmos. 121, 9717-9736 (2016).

37. Zhang, Y., Macke, A. \& Albers, F. Effect of crystal size spectrum and crystal shape on stratiform cirrus radiative forcing. Atmos. Res. 52, 59-75 (1999). 
38. Kärcher, B., Burkhardt, U., Unterstrasser, S. \& Minnis, P. Factors controlling contrail cirrus optical depth. Atmos. Chem. Phys. 9, 6229-6254 (2009).

39. Wilks, D. Statistical Methods in the Atmospheric Sciences. Academic, San Diego, Calif. 467pp. (1995).

40. Unterstrasser, S. \& Gierens, K. Numerical simulations of contrail-to-cirrus transition-part 1: An extensive parametric study. Atmos. Chem. Phys. 10 2017-2036 (2010).

41. Fuglestvedt, J. S. et al. Transport impacts on atmosphere and climate: Metrics. Atmos. Environ. 44, 4648-4677 (2010).

42. Lund M.T. et al. Emission metrics for quantifying regional climate impacts of aviation. Earth System Dynamics 8, 547-563 (2017).

43. Sundqvist, H. A parameterization scheme for non-convective condensation including prediction of cloud water content. Q. J. Roy. Meteor. Soc. 104, 677-690 (1978).

44. Lohmann, U., Spichtinger, P., Jess, S., Peter, T. \& Smit Cirrus, H. cloud formation and ice supersaturated regions in a global climate model. Environ. Res. Lett. 3, 045022 (2008).

45. Burkhardt, U. \& Kärcher, B. Process-based simulation of contrail cirrus in a global climate model. J. Geophys. Res. 114, D16201 (2009).

46. Burkhardt, U., Kärcher, B., Ponater, M., Gierens, K. \& Gettelman, A. Contrail cirrus supporting areas. Geophys. Res. Lett. 35, L16808 (2008).

47. Lamquin, N., Stubenrauch, C., Gierens, K., Burkhardt, U. \& Smit, H. A global climatology for upper-tropospheric ice supersaturation occurence inferred from the atmospheric infrared sounder calibrated by MOZAIC. Atmos. Chem. Phys. 12, 381-405 (2012).
48. Eyers, C. J. et al. AERO2K Global Aviation Emissions Inventories for 2002 and 2025 (QinetiQ Ltd, Farnborough, U.K., 2004). Tech. Rep. QINETIC/04/01113.

49. Schumann U. and A. Heymsfield. On the life cycle of individual contrails and contrail cirrus. Meteorol. Monogr. 2016 https://doi.org/10.1175/ AMSMONOGRAPHS-D-16-0005.1 (2016).

50. Stuber, N., Sausen, R. \& Ponater, M. Stratosphere adjusted radiative forcing calculations in a comprehensive climate model. Theor. Appl. Climatol. 68, 125-135 (2001).

(i) Open Access This article is licensed under a Creative Commons Attribution 4.0 International License, which permits use, sharing, adaptation, distribution and reproduction in any medium or format, as long as you give appropriate credit to the original author(s) and the source, provide a link to the Creative Commons license, and indicate if changes were made. The images or other third party material in this article are included in the article's Creative Commons license, unless indicated otherwise in a credit line to the material. If material is not included in the article's Creative Commons license and your intended use is not permitted by statutory regulation or exceeds the permitted use, you will need to obtain permission directly from the copyright holder. To view a copy of this license, visit http://creativecommons. org/licenses/by/4.0/.

(c) The Author(s) 2018 